\title{
Squeezing enhancement by competing nonlinearities: Almost perfect squeezing without instabilities
}

\author{
C. Cabrillo, J. L. Roldán, and P. García-Fernandez \\ Instituto de Estructura de la Materia, CSIC, Serrano 123, 28006 Madrid, Spain \\ (Received 17 December 1996; revised manuscript received 2 September 1997)
}

\begin{abstract}
The competition between the $\chi^{(2)}$ nonlinearity of a resonant second-harmonic-generation (SHG) system and an added $\chi^{(3)}$ nonlinearity shifts the Hopf bifurcation of the standard SHG towards higher photon numbers eventually completely stabilizing the system. Remarkably, perfect squeezing survives the stabilization. Two important consequences are discussed, namely, efficient bright squeezing generation and strong suppression of the excess noise which, for parameters corresponding to an experiment reported in the literature, can be reduced by two orders of magnitude without diminishing the squeezing. Possible experimental implementations are finally discussed. [S1050-2947(97)05112-3]
\end{abstract}

PACS number(s): 42.50.Dv, 42.50.Lc, 42.65.Ky

From the pioneering "proof of the principle" experiment (only $0.3 \mathrm{~dB}$ of noise reduction) of Slusher et al. [1], the generation of squeezed light has been steadily improving. Thus, in a landmark experiment, Polzik, Carri, and Kimble [2] produced a squeezed vacuum tunable source with $6 \mathrm{~dB}$ of noise suppression, Kim and Kumar [3] a 5.8-dB broadband pulsed squeezed vacuum, and Schiller et al. [4] have been able even of a complete determination of a 5.5-dB squeezed vacuum state. Also, cw bright squeezing has been improving (in noise reduction, output power, and stability) from the early days of Pereira and co-workers [5] while pulsed bright squeezing has also surpassed the $5 \mathrm{~dB}$ figure [6].

All the above-mentioned experiments rely on pure $\chi^{(2)}$ or $\chi^{(3)}$ nonlinear interactions. Given the relative simplicity of these systems, they have enjoyed the favor of both theoreticians and experimentalists. There were, however, some relatively early incursions [7] in more complicated systems combining both kinds of nonlinearities, suggesting enhanced quantum noise reduction. Recently, some theoretical work has confirmed this possibility in two different experimental configurations $[8,9]$. Here, we present a third approach in which the competition between the two nonlinearities is advantageously exploited to enlarge the available power to squeeze the noise as well as to reduce the antisqueezed excess noise. The benefits of competing nonlinearities are not limited only to systems with $\chi^{(2)}$ and $\chi^{(3)}$. Thus, higher nonlinearities could be even better for quadrature squeezing [10], while combining two different kinds of $\chi^{(2)}$ nonlinearities improves the generation of twin beams in nondegenerate parametric oscillators [11].

The system consists of a resonant second harmonic generation configuration with an added intracavity Kerr-like nonlinearity. To specify the model, two modes with frequencies $\omega$ and $2 \omega$, respectively, both resonant in an optical cavity with only one mirror of finite reflectivity, interact with a suitable nonlinear medium characterized by its second $\chi_{2 \omega}^{(2)}$ and third $\chi_{\omega,-\omega, \omega}^{(3)}$ order susceptibilities. An effective interaction Hamiltonian can be written in a suitable rotating frame as $(\hbar=1)$

$$
H_{I}=i \frac{\kappa}{2}\left(a^{\dagger 2} b-a^{2} b^{\dagger}\right)+\frac{\Gamma}{2} a^{\dagger 2} a^{2},
$$

where $\kappa$ and $\Gamma$ are proportional to the $\chi_{2 \omega}^{(2)}$ and $\chi_{\omega,-\omega, \omega}^{(3)}$ susceptibilities, respectively, and $a, b$ are the usual annihilation operators for the fundamental and the second-harmonic modes, respectively. A convenient normalization is achieved by defining

$$
\alpha=\sqrt{\frac{\kappa^{2}}{2 \gamma_{a} \gamma_{b}}} a, \quad \beta=\frac{\kappa}{\gamma_{a}} b,
$$

$\gamma_{a}$ and $\gamma_{b}$ being the total loss rates for the $\omega, 2 \omega$ modes. The classical evolution equations generated by Eq. (1) are

$$
\begin{gathered}
\frac{d \alpha}{d \tau}=-\alpha+\beta \alpha^{*}-i \Lambda \alpha^{*} \alpha^{2}+\lambda, \\
\frac{1}{r} \frac{d \beta}{d \tau}=-\beta-\alpha^{2},
\end{gathered}
$$

where

$$
\tau=\gamma_{a} t, \quad r=\frac{\gamma_{b}}{\gamma_{a}}, \quad \Lambda=\frac{2 \gamma_{b} \Gamma}{\kappa^{2}}, \quad \lambda=\mu_{a} \sqrt{\frac{\kappa^{2}}{\gamma_{b}}} a_{\text {in }},
$$

$a_{\text {in }}$ being the amplitude of an input coherent driving field and $\mu_{a}$ the ratio between the losses at the mirror and the total losses of mode $a$. The concept of squeezing refers to a phase dependence of the quantum noise of an electromagnetic wave so that for some phases the noise level is below that of the vacuum state. This "miracle" can only happen for a finite band of frequencies and therefore the complete description of the phenomenon is accounted for by a phasedependent noise power spectrum. The optimal phase is in general frequency dependent. An analytical optimization of the spectrum on such variable is possible yielding for the maximally squeezed phase and its normal

$$
S_{\mp}(\omega)=1+\mu_{a} S_{\mp}^{N}(\omega),
$$

with 


$$
\begin{aligned}
S_{\mp}^{N}(\omega)= & \frac{1}{2} \int_{-\infty}^{\infty}\left\{\left\langle: a^{\dagger}(\tau+s), a(\tau):\right\rangle\right. \\
& \mp|\langle: a(\tau+s), a(\tau):\rangle|\} e^{i \omega s} d s,
\end{aligned}
$$

where colons denote normal and time ordering and $\omega$ is given in units of $\gamma_{a}$. An equivalent expression is obtained for the $b$ mode. As $S_{-}^{N}(\omega)$ reaches at best $-1, \mu_{a}$ sets the maximum squeezing available to $1-\mu_{a}$. $S_{+}(\omega)$ corresponds to the noise in the perpendicular phase which, by virtue of Heisenberg's principle, shows an excess noise above that of the vacuum which at best equals the noise reduction in the squeezed phase (when measured in $\mathrm{dB}$ with respect to the vacuum noise). Optimizing the squeezing generation is a matter of maximizing the squeezing while minimizing the excess noise. Whenever perfect squeezing is achieved, $S_{+}(\omega)$ must diverge. Such a dominant role of the noise points towards a dynamical instability, and so perfect squeezing can only be reached on them. Strong noise reduction is in this way linked to instabilities, making stability analysis unavoidable. Linearization of Eqs. (2) yields a drift matrix which is formally the same as that given in a previous work [14], since only the inhomogeneous part of the differential equations has been changed. The explicit form for the eigenvalues is [14]

$$
k_{1,2,3,4}=\frac{-r-1+g \mp \sqrt{(-r+1 \pm g)^{2}-8 r\left|\alpha_{f}\right|^{2}}}{2},
$$

where $g=\left|\alpha_{f}\right|^{2} \sqrt{1-3 \Lambda^{2}}$ and $\alpha_{f}$ denotes a fixed point of Eqs. (2). The stability analysis evidences a Hopf bifurcation at $g=r+1$, where $\operatorname{Re} k_{1}=\operatorname{Re} k_{2}=0$ and $\operatorname{Im} k_{1}=-\operatorname{Im} k_{2} \neq 0$. Self-sustained oscillations build up, then, above a critical normalized photon number given by

$$
n_{c} \equiv\left|\alpha_{f}^{c}\right|^{2}=\frac{r+1}{\sqrt{1-3 \Lambda^{2}}}
$$

This critical value reaches infinity at $\Lambda_{\mathrm{th}}=1 / \sqrt{3}$ and, indeed, it can be shown that for larger $\Lambda$ the system is stable. From the above, $n \equiv\left|\alpha_{f}\right|^{2}$ is in units of the photon number at the second-harmonic-generation (SHG) Hopf bifurcation in the limit $r=0$. The efficiencies for each mode (the ratio between the input and the output powers) can be expressed as

$$
\begin{gathered}
\eta_{a}=\frac{\left(2 \mu_{a}-1-n\right)^{2}+n^{2} \Lambda^{2}}{(1+n)^{2}+n^{2} \Lambda^{2}}, \\
\eta_{b}=\frac{4 \mu_{a} \mu_{b} n}{(1+n)^{2}+\Lambda^{2} n^{2}} .
\end{gathered}
$$

As expected, the $\chi^{(3)}$ nonlinearity diminishes the SHG efficiency (from here the term "competing'). Although at a first glance this appears as a disappointing consequence, from the noise reduction perspective, it will turn out to be advantageous.

In order to calculate the quantum average implicit in Eq. (4) we follow the standard procedure first introduced in [12]. This method relies on a linearization of the evolution equations which fails near the instabilities and so, assertions such

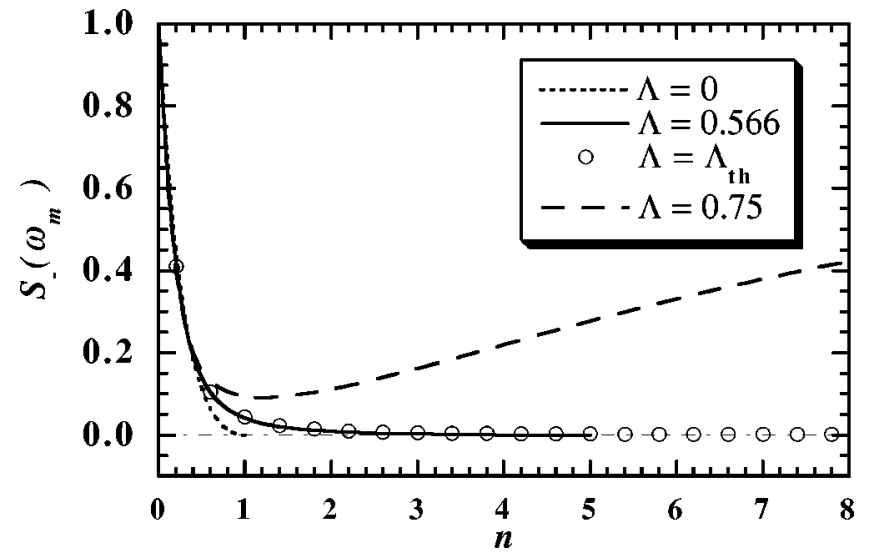

FIG. 1. The maximum squeezing in the fundamental mode as a function of the photon number for the ideal case $r=10^{-6}$ and $\mu_{a}=1 . \Lambda=0.566$ corresponds to $n_{c}=5$.

as "perfect squeezing" must be understood with some caution. However, more elaborate procedures [13] show that the method only breaks down rather close to instabilities, making it a reliable guide for squeezing prospection. When no Kerr effect is present, perfect squeezing can be reached at the critical point in the limits of total asymmetry in the losses [12] ( $r \rightarrow 0$ for perfect squeezing in the fundamental, $r \rightarrow \infty$ for the harmonic mode). In order to study how the squeezing is affected by the third-order nonlinearity, we use the maximum squeezing available for fixed values of $n, \Lambda$, and $r$ [denoted by $S_{-}\left(\omega_{m}\right)$ ] which is obtained by numerical optimization of $S_{-}(\omega)$ with respect to the frequency. We first focus on an ideal case with $\mu_{a}=1$ and $r=10^{-6}$ as a numerical approximation to the limit case $r=0$. Figure 1 shows the evolution of $S_{-}\left(\omega_{m}\right)$ with increasing $\Lambda$. The curve for $\Lambda=0.566$ strongly suggests that perfect squeezing can also be reached at the corresponding critical point $\left(n_{c}=5\right)$. Indeed, this is the case for any $n_{c}$ in the limit $r=0$, as will be shown elsewhere. The noise suppression increases so fast with $n$ that, in practice, almost perfect squeezing [say, $\left.S\left(\omega_{m}\right)<0.05\right]$ is reached from $n=3$ on. Most remarkably, the same dependence on $n$ remains till $\Lambda_{\text {th }}$, for which there is no instability. In other words, the conventional wisdom linking strong noise suppression to the proximity to an instability is shattered into pieces. The importance of this result goes beyond the particular system proposed here, as it opens the possibility of high squeezing free from the delicacy of working points close to instabilities. Theoretical studies aimed to clarify the general conditions under which this can happen are in progress. The highly nontrivial behavior of the system below $\Lambda_{\text {th }}$ is also highlighted by the fact that the squeezing (and so the quantum character of the state) increases monotonically with the photon number (quantum number), just in counterposition to a naive application of the correspondence principle. It is worth noticing that essentially the same plots as in Fig. 1 are obtained till $r=0.01$, not such an unthinkable value.

Turning now to a more realistic case we choose a mild asymmetry of $r=0.15$ and a conservative $\mu_{a}=0.9$. The behavior of the system is summarized in Fig. 2, showing both $S_{-}\left(\omega_{m}\right)$ and $S_{+}\left(\omega_{m}\right)$ (in dB's relative to the vacuum noise), as well as the "efficiency" $\eta_{a}$. Now, the squeezing even increases above the standard SHG when $\Lambda$ is set to $\Lambda_{\text {th }}$. The excess noise, however, grows up disappointingly fast with $n$. 


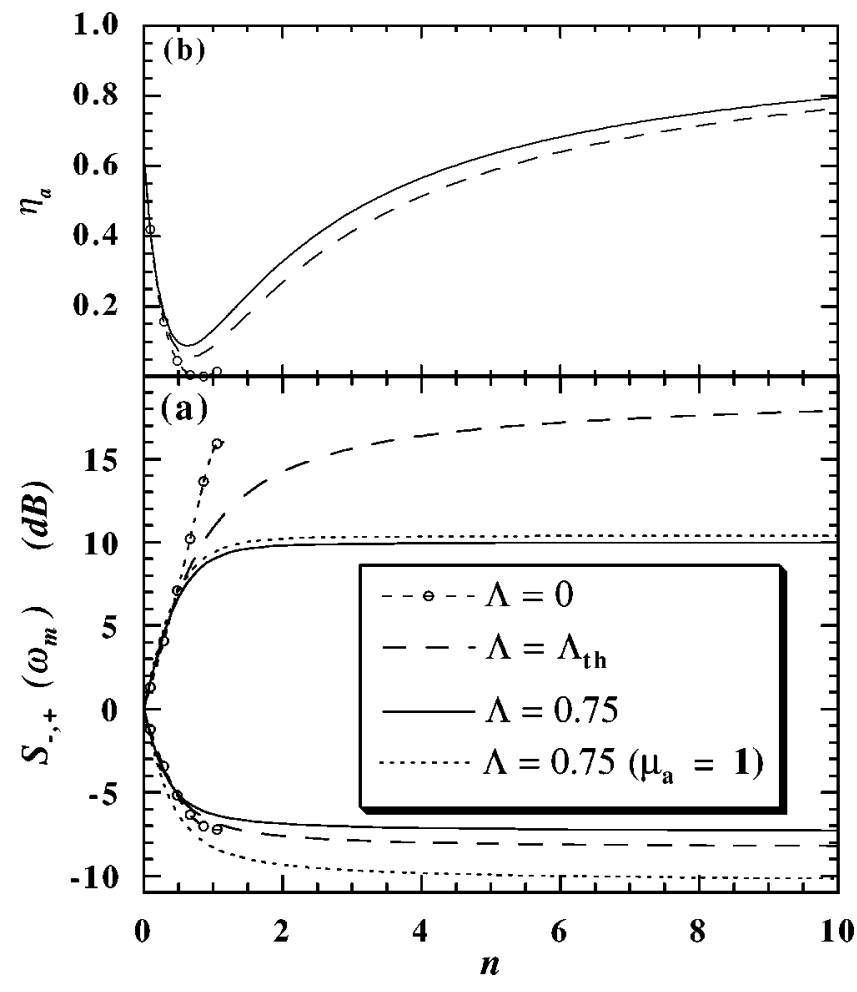

FIG. 2. The maximum noise reduction and the excess noise (fundamental mode) for $r=0.15$ and $\mu_{a}=0.9$ (a) and the corresponding $\eta_{a}$ efficiency (b).

Still, there is a significant reduction around $n=1.4$, where the $\Lambda_{\text {th }}$ case equals the maximum of the standard SHG. Fortunately, increasing $\Lambda$ the excess noise decreases dramatically while the squeezing diminishes slowly. Thus, for $\Lambda=0.75$ the squeezing still is very high, reaching the maximum of the SHG case around $n=10$ and even surpassing it above this $n$, while the excess noise is strongly suppressed (around $6 \mathrm{~dB}$ ). Indeed, setting $\mu_{a}=1$ the excess noise shows an almost symmetric behavior with respect to the squeezing, the signature of a Heisenberg limited performance. Interestingly enough, $\eta_{a}$ increases above a certain minimum located below the SHG Hopf bifurcation. In other words, the system presents unique properties as a quantum noise eater, namely, any increase of the input power above the mentioned minimum amounts in both a higher squeezing and a higher efficiency in the output power generation. In contrast, in the standard SHG system the maximum squeezing almost coincides with the minimum of the power efficiency. Except for the increasing power efficiency, the performance of the system is essentially the same for the harmonic mode when $r>1$.

The previous results, although certainly interesting, underestimate the performance of the system. Whether $S_{-}\left(\omega_{m}\right)$ reaches zero or not, at $n_{c}$ the noise must diverge at some frequency. Once perfect squeezing is not achieved such a frequency does not necessarily coincide with $\omega_{m}$. The whole spectrum is then necessary to completely characterize the noise behavior of the system. Figure 3 illustrates the point very vividly. The parameter values have been chosen to reproduce the theoretical curve of the experiment of Kurz et al. [16] in order to make contact as much as possible with a real situation. The performance of the system is amazingly supe-

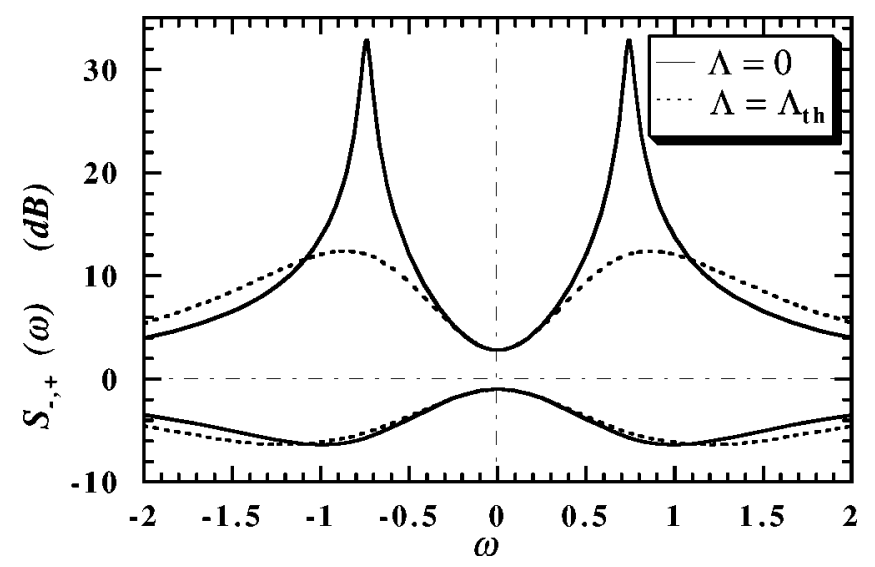

FIG. 3. The effect of $\chi^{(3)}$ on $S_{-}(\omega)$ and $S_{+}(\omega)$ for the fundamental mode; $r=0.25, \mu_{a}=0.91$, and $n=1.2$.

rior when the $\chi^{(3)}$ nonlinearity is introduced. The suppression of the excess noise is huge (around $22 \mathrm{~dB}$ ) without any degradation of the squeezing. Equally high (from 27 to 6.5 $\mathrm{dB})$ is the reduction of excess noise in the harmonic mode. One can reasonably expect a mitigation of the severe technical problems found in [16] after such a suppresion of noise.

From the definitions of $\kappa, \Gamma$, and $\gamma_{b}$ and assuming planewave modes in the resonator and perfect phase matching, it can be shown that $\Lambda=\left(3 T_{b} / 8 \pi\right)\left(\lambda_{a} / l\right)\left[n_{r} \chi^{(3)} /\left(\chi^{(2)}\right)^{2}\right]$, with $T_{b}$ being an effective transmission including all the mirrors as well as the scattering and absorption losses for mode $b, n_{r}$ the refraction index, and $\lambda_{a}$ the wavelength of mode $a$. The most obvious way of practical implementation of the system would be to place two different materials in the same cavity, one suited for the second order interaction, the other for the third. However, for the low values of $\Lambda$ needed, a simple crystal could be adequate for the task. For example, from the KDP electro-optic Kerr coefficients $\left(s_{66}=0.9 \times 10^{-18} \mathrm{~m}^{2} / \mathrm{V}^{2}[15]\right)$, a $\chi_{0,0, \omega}^{(3)}$ of $1.1 \times 10^{-18} \mathrm{~m}^{2} / \mathrm{V}^{2}$ at $512 \mathrm{~nm}$ is obtained. As a first approximation we shall assume $\chi_{0,0, \omega}^{(3)}=\chi_{\omega,-\omega, \omega}^{(3)}$. Although the approximation may seem crude, it gives the right order of magnitude for liquid $\mathrm{CS}_{2}$, for which both kinds of Kerr nonlinearities are reported in the literature [15]. Taking $\chi_{2 \omega}^{(2)}=10^{-12} \mathrm{~V} / \mathrm{m}$ [15], $T_{b}=0.03, \lambda_{a}=512 \mathrm{~nm}$, and $l=0.5 \mathrm{~cm}, \Lambda$ equals $\Lambda_{\text {th }}$. Power requirements are, however, quite strong with KDP but sensible figures are still possible. For instance, for $r=0.5$, $\mu_{a}=0.9$, and $n=1.44$ (the same proportion with respect to $n_{c}$ as in [16]) the squeezing is around $-5 \mathrm{~dB}$, while the maximum excess noise in the standard SHG is around $33 \mathrm{~dB}$. With only a $\Lambda=0.3\left(T_{b} \simeq 0.016\right.$, letting the rest of the parameters remain unchanged) this excess noise is reduced by $13 \mathrm{~dB}$. Assuming a waist of the beam of $33.5 \mu \mathrm{m}$ (as in [16]), plane modes (with a section of radius $\sqrt{2}$ times the waist), and perfect phase matching we estimate the input power necessary to reach $n=1.44$ in $195 \mathrm{~mW}$, quite a sensible value. All of this is admittedly oversimplified. For instance, depending on the values of the $\chi^{(3)}$ tensor components and the losses, the self-phase modulation of the $b$ mode and the cross phase modulation could be important, although not necessarily deleterious. The previous analysis must be therefore be considered only as a preliminary plausibility argument.

A second very interesting approach is the use of asym- 
metric multiple quantum wells. These devices are currently the object of an intense study regarding their applicability for SHG [17]. For our purposes, they are excellent candidates, as the energy-level schemes used for $\chi^{(2)}$ optimization are, at the same time, adequate for $\chi^{(3)}$ enhancement. Even more, the nonlinearities can be controlled with an applied dc field. A drawback of such systems is the increased absorption associated with the resonant enhancement of nonlinearities. The augmented total losses could force the use of pulsed light so that the results shown here would not be directly applicable. However, as we are not interested in a giant $\chi^{(2)}$ but in a compensated value of $n_{r} \chi^{(3)} /\left(\chi^{(2)}\right)^{2}$, resonance is not so critical and maybe an adequate working point with a sufficiently diminished absorption could be found.

Cascaded second-order nonlinearities (see, for instance, [18] for an experimental demonstration of dispersive bistability by cascading effect) are also very interesting since the $\chi^{(2)}$ and the effective $\chi^{(3)}$ are, as in the previous cases, naturally embedded in the same material.

Poled fibers [19] which generate a SHG signal would be a technologically very attractive possibility. Although pulses are in principle needed in order to achieve an appreciable self-phase modulation, maybe the cascading effect could help to enable $\mathrm{cw}$ operation.

Finally, the stabilization induced by the $\chi^{(3)}$ nonlinearity could be useful also at the classical level as it makes far more accessible the point of maximum SHG efficiency (the minimum of $\eta_{a}$ ).

This work was supported in part by Project No. TIC950563-C05-03 (CICYT, Spain). C.C. wishes to thank S. Schiller, C. Fabre, and F. Agulló-López for useful conversations and R. Serna for providing valuable bibliographic and nonlinear materials data.
[1] R. E. Slusher et al., Phys. Rev. Lett. 55, 2409 (1985).

[2] E. S. Polzik, J. Carri, and H. J. Kimble, Appl. Phys. B: Photophys. Laser Chem. 55, 279 (1992).

[3] C. H. Kim and P. Kumar, Phys. Rev. Lett. 73, 1605 (1994).

[4] S. Schiller et al., Phys. Rev. Lett. 77, 2933 (1996).

[5] S. F. Pereira et al., Phys. Rev. A 38, 4931 (1988); A. Sizmann et al., Opt. Commun. 80, 138 (1990); P. Kurz et al., Europhys. Lett. 24, 449 (1993); R. Paschotta et al., Phys. Rev. Lett. 72, 3807 (1994); H. Tsuchida, Opt. Lett. 20, 2240 (1995).

[6] K. Bergman et al., Opt. Lett. 19, 290 (1994).

[7] P. Tombesi and H. P. Yuen, in Coherence and Quantum Optics V, edited by L. Mandel and E. Wolf (Plenum, New York, 1984); P. Tombesi, in Quantum Optics IV, edited by J. D. Harvey and D. F. Walls (Springer, Berlin, 1986).

[8] C. Cabrillo and F. J. Bermejo, Phys. Rev. A 48, 2433 (1993).

[9] K. Sundar, Phys. Rev. Lett. 75, 2116 (1995).

[10] P. Tombesi and A. Mecozzi, Phys. Rev. A 37, 4778 (1988); P. Tombesi, ibid. 39, 4288 (1989).
[11] M. A. M. Marte, Phys. Rev. Lett. 74, 4815 (1995); J. Opt. Soc. Am. B 12, 2296 (1995).

[12] M. J. Collet and D. F. Walls, Phys. Rev. A 32, 2887 (1985).

[13] C. J. Mertens, T. A. B. Kennedy, and S. Swain, Phys. Rev. A 48, 2374 (1993); Phys. Rev. Lett. 71, 2014 (1993); C. J. Mertens and T. A. B. Kennedy, Phys. Rev. A 53, 3497 (1996).

[14] C. Cabrillo and F. J. Bermejo, Phys. Lett. A 170, 300 (1992).

[15] F. Agulló-López, J. M. Cabrera, and F. Agulló-Rueda, Electrooptics: Phenomena, Materials and Applications (Academic, New York, 1994).

[16] P. Kurz et al., Europhys. Lett. 24, 449 (1993).

[17] C. Sirtori et al., Appl. Phys. Lett. 60, 151 (1992); S. Janz, F. Chatenoud, and R. Normandin, Opt. Lett. 19, 622 (1994); V. Milanovic and Z. Ikonic, IEEE J. Quantum Electron. 32, 1316 (1996); I. Vurgaftman et al., ibid. 32, 1334 (1996).

[18] A. G. White, J. Mlynek, and S. Schiller, Europhys. Lett. 35, 425 (1996).

[19] P. G. Kazansky, L. Dong, and P. St. J. Russell, Opt. Lett. 19, 701 (1994) 